\title{
Elementary excitations of the BCS model in the canonical ensemble
}

\author{
José María Román, ${ }^{1}$ Germán Sierra, ${ }^{1}$ and Jorge Dukelsky ${ }^{2}$ \\ ${ }^{1}$ Instituto de Física Teórica, CSIC/UAM, Madrid, Spain \\ ${ }^{2}$ Instituto de Estructura de la Materia, CSIC, Madrid, Spain
}

(Received 1 August 2002; published 28 February 2003)

\begin{abstract}
We have found the elementary excitations of the exactly solvable BCS model for a fixed number of particles. These turn out to have a peculiar dispersion relation, some of them with no counterpart in the Bogoliubov picture, and unusual counting properties related to an old conjecture made by Gaudin. We give an algorithm to count the number of excitations for each excited state and a graphical interpretation in terms of paths and Young diagrams. For large systems the excitations are described by an effective Gaudin model, which accounts for the finite-size corrections to BCS.
\end{abstract}

DOI: 10.1103/PhysRevB.67.064510

\section{INTRODUCTION}

The paradigmatic model to study the superconducting properties of metals ${ }^{1}$ and nuclei $^{2}$ is the pairing model proposed by Bardeen, Cooper, and Schrieffer. The ground state (GS) and excitations of the BCS model are well known in the grand canonical (g.c.) ensemble, and explain the behavior of systems with large numbers of particles. However, for small systems, such as nuclei or nanograins, one is forced to work with a fixed number of particles, where the g.c. BCS wave function, including its projected version, are not adequate. The problem is due to the strong pairing fluctuations, with which a mean-field approach cannot deal properly. An alternative approach is provided by exact numerical methods, as the density-matrix renormalization-group, ${ }^{3}$ but their complexity somehow obscures the physics behind. Fortunately enough the reduced BCS model, characterized by a unique pairing coupling $g$, is exactly solvable, as was shown by Richardson and Sherman. ${ }^{4}$ This exact solution has been recently used in connection with superconducting nanograins (see Ref. 6 for a review). ${ }^{5}$

Most of the exact studies deal with the GS and the excited states that are obtained by breaking Cooper pairs. However, one must also consider the promotion of pairs to higher energy levels (bosonic pair-hole excitations). This paper focuses on the latter type of excitations since the former ones can be easily included into our formalism. We shall indicate the peculiar dynamics and the unusual counting properties exhibited by the excitations of the exactly solvable BCS model, some of them with no analog in the standard picture of Bogoliubov quasiparticles. These features account for the exact finite-size corrections to the thermodynamic limit, obtained from the standard BCS treatment.

In Sec. II we introduce the superconducting system described by Richardson and Gaudin models, and we present here our conjecture about what represents their elementary excitations. Section III is devoted to classifying the excited states according to what will be interpreted as elementary excitations in Sec. IV. In this respect a diagramatic representation of the excited states turns out to be very useful. The thermodynamic limit of our theory is presented in Sec. IV, which confirms our conjecture about the elementary excitations. A comparison with the BCS theory is also presented.
Finally, Sec. V contains our conclusions.

\section{RICHARDSON AND GAUDIN MODELS}

Let us consider a fermionic system with $N$ single-particle energy levels. The reduced BCS Hamiltonian decouples the levels which are singly occupied and one is left with those that are either empty, $|0\rangle$ (hole), or doubly occupied, $b_{j}^{\dagger}|0\rangle$ (pair), with an energy $\varepsilon_{j}\left(b_{j}^{\dagger}=c_{j,+}^{\dagger} c_{j,-}^{\dagger}\right.$ is a hard-core boson operator that creates a pair of two time-reversal states). We shall suppose that the singly occupied levels have been removed. Since the latter ones decouple, their effect can be considered easily by adding their free energy $\varepsilon_{j}$ to the total energy. The complete system will be treated elsewhere. The reduced BCS Hamiltonian reads

$$
H_{B C S}=\sum_{j=1}^{N} \varepsilon_{j} b_{j}^{\dagger} b_{j}-G \sum_{j, j^{\prime}=1}^{N} b_{j}^{\dagger} b_{j^{\prime}},
$$

where $G$ is a dimensionful coupling constant. The standard model employed to study nanograins is given by the choice $\varepsilon_{j}=d(2 j-N-1)$, where $d=\omega / N$ is the single-particle energy-level spacing and $\omega / 2$ is the Debye energy. ${ }^{6}$ The coupling $G$ can be written as $G=g d$, where $g$ is dimensionless. The value of the bulk BCS gap, $\Delta_{B C S}$, of the equally spaced model is given by $\Delta_{B C S}=\Delta / 2$, where $\Delta=\omega / \sinh (1 / g)$.

The eigenstates of Eq. (1) with $M$ pairs are given by ${ }^{4}$

$$
\left|\left\{E_{\mu}\right\}_{\mu=1}^{M}\right\rangle=\prod_{\mu=1}^{M} B_{\mu}^{\dagger}|0\rangle, \quad B_{\mu}^{\dagger}=\sum_{j=1}^{N} \frac{b_{j}^{\dagger}}{\varepsilon_{j}-E_{\mu}},
$$

where the parameters $\left\{E_{\mu}\right\}_{\mu=1}^{M}$ satisfy the Richardson equations

$$
\frac{1}{G}=\sum_{j=1}^{N} \frac{1}{\varepsilon_{j}-E_{\nu}}-\sum_{\mu \neq \nu}^{M} \frac{2}{E_{\mu}-E_{\nu}}, \quad \nu=1, \ldots, M .
$$

The total energy of the state (2) reads $E=\sum_{\mu=1}^{M} E_{\mu}$. The number of solutions of Richardson Eqs. (3) is given by the binomial coefficient, $C_{M}^{N}=\left(\begin{array}{l}N \\ M\end{array}\right)$, and coincides with the dimension of the Hilbert space, $\mathcal{H}_{M}^{N}$, of states with $M$ pairs distributed into $N$ different levels. Then it is natural to label 
TABLE I. Classification of roots in the $g \rightarrow \infty$ limit.

\begin{tabular}{ccccc}
\hline \hline Number of solutions & $d_{M}$ & $d_{M-1}$ & $d_{1}$ & $d_{0}=1$ \\
\hline$E_{\mu}$ finite & $M$ & $M-1$ & 1 & 0 \\
$E_{\mu}$ infinite & 0 & 1 & $M-1$ & $M$ \\
\hline \hline
\end{tabular}

Richardson states by a set of integers $I=\left\{j_{1}, \ldots, j_{M}\right\}$ corresponding to the value that the pair energies $E_{\mu}$ take at $g$ $=0$, i.e., some of the $\varepsilon_{j}$ 's.

The BCS model can be mapped into a spin system which at $g \rightarrow \infty$ has $\mathrm{SU}(2)$ symmetry. Based on this fact Gaudin ${ }^{7}$ made the conjecture that given a solution $\left\{E_{\mu}(g)\right\}_{\mu=1}^{M}$ of Eqs. (3), and taking the limit $g \rightarrow \infty$, a subset of them, say, $\left\{E_{\alpha}(\infty)\right\}_{\alpha=1}^{N_{G}}$, remain finite and satisfy the equations

$$
0=\sum_{j=1}^{N} \frac{1}{\varepsilon_{j}-E_{\alpha}}-\sum_{\beta \neq \alpha}^{N_{G}} \frac{2}{E_{\beta}-E_{\alpha}}, \alpha=1, \ldots, N_{G},
$$

while the remaining $M-N_{G}$ roots tend to infinity and satisfy Eqs. (3) with all $\varepsilon_{j}$ 's set to zero. The number $N_{G}$ of finite roots takes values from 0 to $M$. The number of solutions of Eqs. (4) is given by $d_{N_{G}}=C_{N_{G}}^{N}-C_{N_{G}-1}^{N} \cdot{ }^{7}$ Therefore in the large $g$ limit the $C_{M}^{N}$ Richardson's solutions would be classified in terms of $N_{G}$ according to Table I. Consistency is guaranteed by the equation $C_{M}^{N}=\Sigma_{N_{G}=0}{ }^{M} d_{N_{G}}$.

We show in this paper that Gaudin finite energies represent the elementary excitations of the superconducting system in the canonical ensemble. Their peculiar dispersion relations and the unusual counting properties will account for the finite-size corrections to the mean-field BCS treatment of superconductivity.

This result is motivated by the excitation energy for large values of $g$, namely, $E_{e x c} \equiv E-E_{G S} \sim g \omega N_{G}\left[1-\left(N_{G}\right.\right.$ $-1) / N]$, and the gap $\Delta \sim g \omega$. Thus, in the large $N$ limit the excitation energy goes as $E_{\text {exc }} \sim N_{G} \Delta$, which allows us to think of the state as a set of $N_{G}$ elementary excitations contributing each with an energy $\Delta$ to the total energy.
In Sec. IV we extend this result for the whole range of $g$. In the meanwhile the next section is devoted to the proof of Gaudin's conjecture given in Table I. We also obtain a formula which gives the number of finite Gaudin energies $N_{G}$ for a given Richardson configuration $I$, and therefore the number of elementary excitations.

\section{CLASSIFICATION OF EXCITED STATES}

\section{A. Simple examples}

Let us first consider the simplest examples given by the excited states with one and two energies remaining finite, i.e., $N_{G}=1$ and 2 . Representatives of these, together with the GS, are shown in Fig. 1, which depicts the real part of the energies, and in Fig. 2, which shows the distribution of the energies in the complex plane for $g=1.5$ and a system with $M=20$ pairs at half filling, i.e., $N=2 M$. As a general feature we see that for small $g$ all parameters $E_{\mu}$ are real, and as $g$ grows some of them collapse and become complexconjugate pairs, which share their real part (this corresponds to two curves merging into a single one in Fig. 1). Figure 2 shows how the energies $E_{\mu}$ arrange themselves into an arc which opens up to infinity as $g \rightarrow \infty$.

The state of Fig. 1(a) corresponds to the GS of the system, and it is labeled by $I_{0}=\{1,2, \ldots, M\}$, which at $g=0$ is identical to the Fermi state (FS). As $g \rightarrow \infty$ all the roots become complex and escape to infinity. According to Table I, this is the only state where this may happen, hence $N_{G}\left(I_{0}\right)=0$.

The lowest excited state $I_{1}=\{1, \ldots, M-1, M+1\}$ is shown in Fig. 1(b). The last root $E_{M}$, which is equal to $\varepsilon_{M+1}$ at $g=0$, stays finite as $g \rightarrow \infty$, while the remaining $M-1$ roots go to infinity, thus $N_{G}\left(I_{1}\right)=1$. All the states with $N_{G}=1$ can be obtained from the FS by (i) promoting the nearest pair below the Fermi level (FL) into one of the $N-M$ empty levels above it, or (ii) moving the nearest hole above the FL into one of the $M$ occupied levels below it. The state $I_{1}$ can be obtained in both ways. Hence the number of $N_{G}=1$ excited states is $d_{1}=(N-M)+M-1$.

The state of Fig. 1(c), $I_{2}=\{1, \ldots, M-2, M, M+2\}$, has $N_{G}\left(I_{2}\right)=2$. All the states with $N_{G}=2$ can be obtained

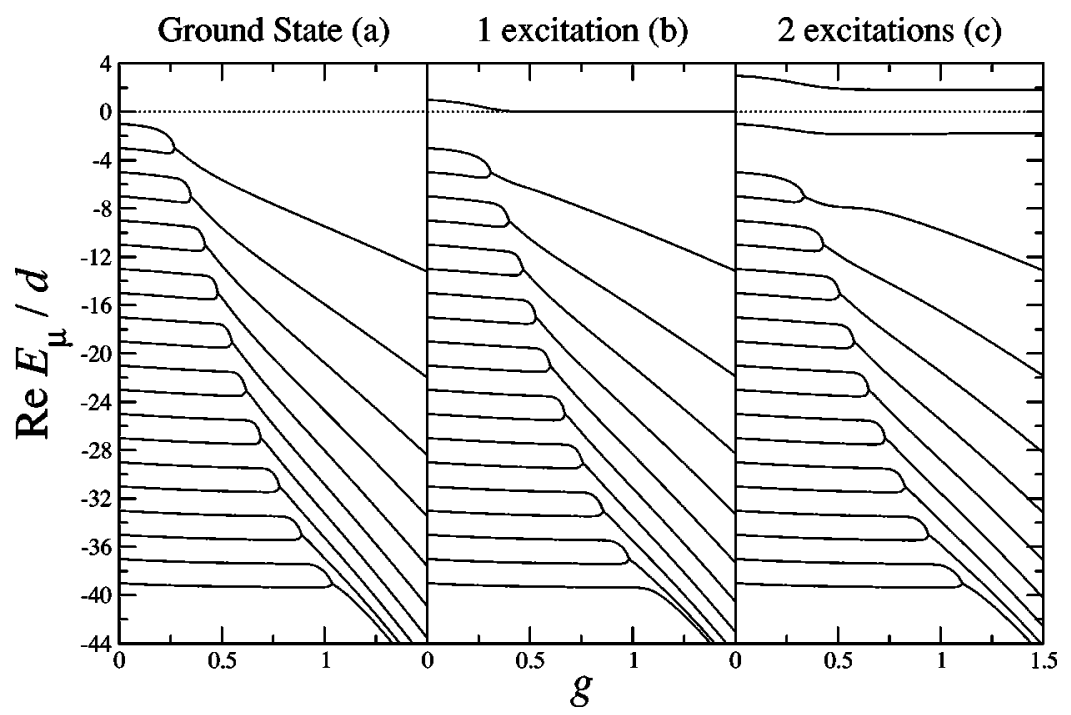

FIG. 1. Real part of $E_{\mu}$ for the equally spaced model with $M=N / 2=20$ pairs and $N_{G}=0,1$, and 2 excitations. 
by the following three different ways from the FS: (i) moving the two pairs just below the FL into the $N-M$ empty levels ( $C_{2}^{N-M}$ states), (ii) moving the two holes just above the FL into the $M$ occupied levels ( $C_{2}^{M}$ states), or (iii) moving one of the $M-1$ pairs in the FS, except the closest to the FL, into one of the $N-M-1$ vacancies above the FL, except the closest to the FL $[(N-M-1)(M-1)$ states $]$. The state with two holes just below and two pairs just above the FL is generated by (i) and (ii), thus the number of states is the expected one, $d_{2}=C_{2}^{N-M}+C_{2}^{M}+(N-M-1)(M-1)$ -1 . This example shows that the value of $N_{G}$ for a generic state depends dramatically on the arrangement of holes and pairs around the FL.

\section{B. $N_{G}(I)$ formula}

We now turn to the evaluation of $N_{G}(I)$ for a general state. One naively expects that this formula should be given by the sum of pairs, $N_{p}$, and holes, $N_{h}$, above and below the FL, respectively, i.e., $N_{G}(I)=N_{p}+N_{h}$. In fact $N_{p}=N_{h}$ since every pair above the FL comes from a hole below it. However this ansatz does not always work as we have already seen above. For example, according to this formula, the state $I_{1}$ of Fig. 1(b) would have 2 instead of $N_{G}=1$, while the state $I_{2}$ of Fig. 1(c) has $N_{G}=2$, which is the correct value.

Let us introduce for convenience the occupation representation of the states $I$, where a pair, a hole, and the FL are depicted as $\bullet, \bigcirc$, and $\mid$, respectively. In the cases discussed above we obtain $I_{0}=\mathbf{0} \cdots 0 \bigcirc \bigcirc \bigcirc, \quad I_{1}$ $=\bullet \cdots \bullet \bigcirc \bigcirc \bigcirc \cdots \circ$, and $I_{2}=\cdots \bullet \bigcirc \vee \bigcirc$ ...

We have found an algorithm to compute $N_{G}(I)$. Given an integer $\ell \geqslant 0$, let us split $I$ into three disjoint sets, $I$ $=A_{\ell} \cup B \cup C_{\ell}$, where $A_{\ell}$ contains the lowest $M-\ell$ levels, $B_{\ell}$ the next $2 \ell$ levels, and $C_{\ell}$ the remaining $N-M$ $-\ell$ ones. For $\ell=0$, the set $B_{0}$ is empty, and $A_{0}$ (respectively, $C_{0}$ ) contains all the levels below (respectively, above) the FL, while for $\ell \geqslant 1$ the set $B \ell$ contains the nearest $\ell$ levels above and below the FL. As an example, let us choose

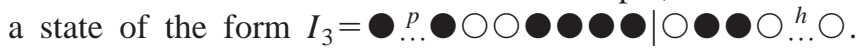

For $\ell=2$ the partition of $I_{3}$ is given by $\left\{\bigcirc{ }^{p} \bigcirc \bigcirc \bigcirc \bigcirc \bigcirc\right\}\{\bigcirc \mid \bigcirc \bigcirc\}\left\{\bigcirc{ }^{h} \bigcirc\right\}^{3}$.

Let us define the number of pairs and holes for each set, i.e., $N_{p / h}^{X}\left(X=A_{\ell}, B_{\ell}, C_{\ell}\right)$. The algorithm giving $N_{G}(I)$ is

$$
\begin{gathered}
N_{G}(I)=\min { }_{\ell=0, \ldots, 2 N_{p}} N_{G}(I, \ell), \\
N_{G}(I, \ell) \equiv N_{h}^{A \ell}+\min \left(N_{h}^{B} \ell, N_{p}^{B} \ell\right)+N_{p}^{C_{\ell}} .
\end{gathered}
$$

Applying this formula to $I_{3}$ one gets $\left\{N_{G}\left(I_{3}, \ell\right)\right\}_{\ell=0}^{4}$ $=\{4,5,4,3,4\}$ and thus, $N_{G}\left(I_{3}\right)=3$. The value of $\ell_{I}$, which minimizes $N_{G}(I, \ell)$, is given in this case by 3 [in general $\ell_{I}$ is not equal to $\left.N_{G}(I)\right]$. The result of this formula is bounded, $N_{p} \leqslant N_{G} \leqslant 2 N_{p}$, and therefore any state with a finite $N_{p}$ would contain a finite number of Gaudin energies. The uncorrelated counting formula proposed earlier coincides with the case $\ell_{I}=0$, since $N_{G}(I, 0)=N_{p}+N_{h}$ (notice that $N_{h}$ $=N_{h}^{A_{0}}$ and $N_{p}=N_{p}^{C_{0}}$ ).

The physical mechanism underlying Eq. (5) is the collective behavior of the holes and pairs that occupy the $\ell_{I}$ closest levels to the FL. In a certain sense, $\ell_{I}$ measures the range of correlation involved in the creation of the elementary excitations out of an initial pair-hole configuration. However, this correlation can be lifted introducing a shifted Fermi level $F L\left(\ell_{I}\right)$ defined by moving the FL an amount of $\ell_{I}$ levels downwards (respectively, upwards) whenever $N_{p}^{B} \ell$ is lower or equal (respectively, greater) than $N_{h}^{B} \ell$. This new Fermi level defines a new Fermi state out of which the excited state with $N_{G}$ finite energies is obtained by the creation of uncorrelated pairs above and holes below the $F L\left(\ell_{I}\right)$. This construction provides a pathway to the g.c. formulation as discussed later.

The formula presented here allows us to prove Gaudin's conjecture by looking for all the states with a given $N_{G}$, and finding out that their number corresponds to $d_{N_{G}}$ as stated in Table I, the same way we already did with those with $N_{G}$ $=1$ and 2 .

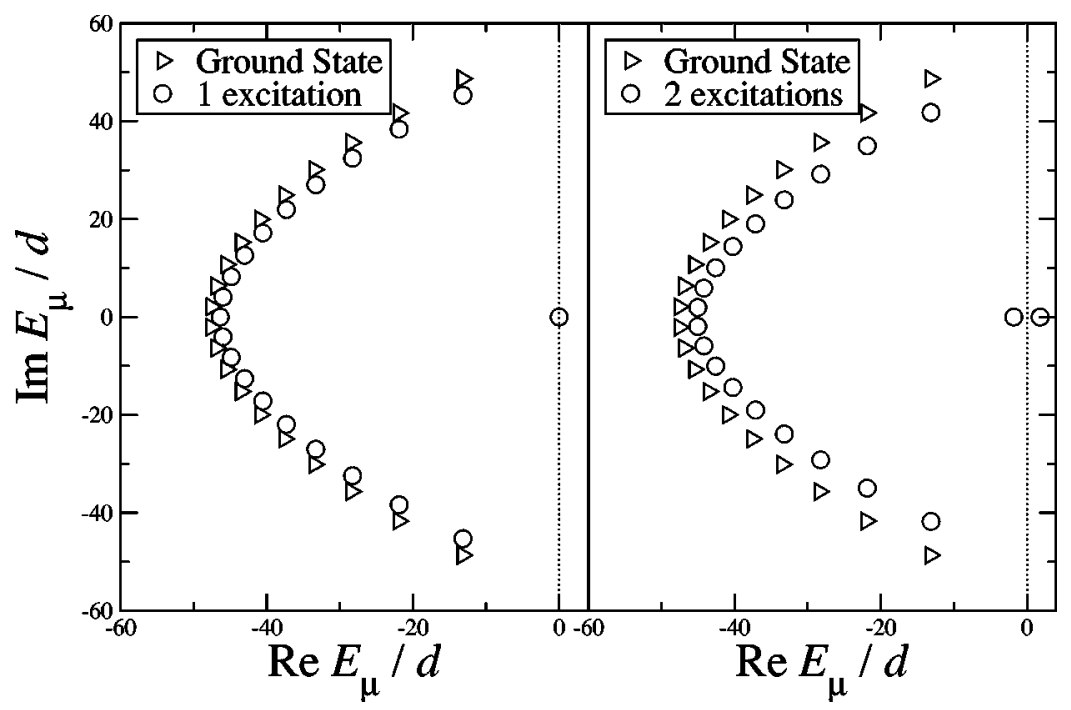

FIG. 2. Position of the $M=20$ pairs of the states of Fig. 1 at $g=1.5$. The arcs $\Gamma_{I_{1}}$ (19 pairs) and $\Gamma_{I_{2}}$ (18 pairs) are a slight modification of the GS $\operatorname{arc} \Gamma_{I_{0}}(20$ pairs $)$. 

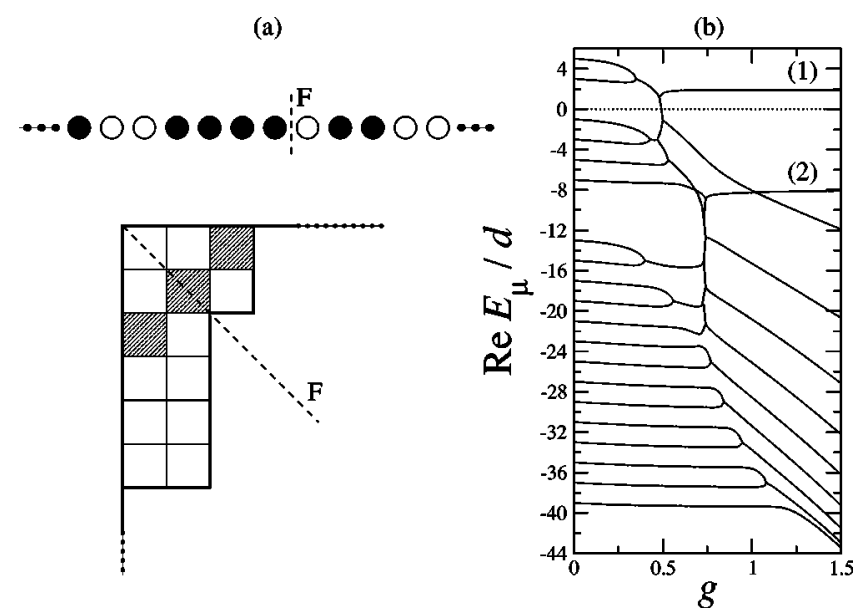

FIG. 3. (a) The path and Young diagram of $I_{3}$. (b) Real part of $E_{\mu}$ for $I_{3}$. For $g$ large enough there is a real root (1) and a complex root (2).

\section{Young diagrams representation}

The correlated behavior of the excitations is made more explicit by a pictorial representation of the states. The idea is to associate to every set $I$ a path $\gamma_{I}$ with $N$ links on the square lattice $\mathcal{Z}^{2}$, starting at the origin $(0,0)$. This is achieved by associating a horizontal link directed to the right, to every hole $\bigcirc$, and a vertical link directed upwards, to every pair . The map starts from the lowest-energy level and ends at the highest one. For example, the path associated to the Fermi state $I_{0}={ }^{M} \bigcirc \mid \bigcirc^{N-M} \bigcirc$ is a polygonal line joining the points $(0,0) \rightarrow(0, M) \rightarrow(N-M, M)$. If $I$ describes a state with $M$ pairs and $N$ energy levels, then the path $\gamma_{I}$ ends at the point $(N-M, M)$. The number of these sort of paths is $C_{M}^{N}$, which is precisely the dimension of the Hilbert space $\mathcal{H}_{M}^{N}{ }^{8}$

In Fig. 3(a) we depict the occupation and path representations of the state $I_{3}$ which yields $N_{G}=3$, in agreement with the numerical results shown in Fig. 3(b). Moreover, Fig. 3(a) illustrates the fact that any state $I$ gives rise to a Young diagram (YD) $Y_{I}$, whose boundary is formed by the links which belong either to $\gamma_{I}$ or to $\gamma_{I_{0}}$, but not to both. The YD of the Fermi state is by construction empty, i.e., $Y_{I_{0}}=\emptyset$. These YD's capture the basic properties of the excitations. First of all, $N_{G}(I)$, given by Eq. (5), coincides with the number of squares on the longest southwest-northeast(SW-NE) diagonal on $Y_{I}$ [see Fig. 3(a)]. This fact provides a geometrical meaning to $N_{G}(I)$ and leads to a combinatorial proof of Gaudin's conjecture, which can be stated as follows: $d_{N_{G}}$ is the number of YD's, $Y_{I}$, associated to the paths $\gamma_{I}$, which have $N_{G}$ squares on their longest SW-NE diagonal. The proof of this conjecture uses the methods of Ref. 8. ${ }^{9}$ This result serves to classify the excitations in terms of YD's. For example, the states with $N_{G}=1$ and 2 discussed above correspond to the YD's shown in Fig. 4.

Other properties of these diagrams are (i) the pair-hole transformation of the states induces a transposition of their associated YD's, (ii) the main northwest-southeast diagonal on a YD coincides with the FL [see Fig. 3(a)], and (iii) the

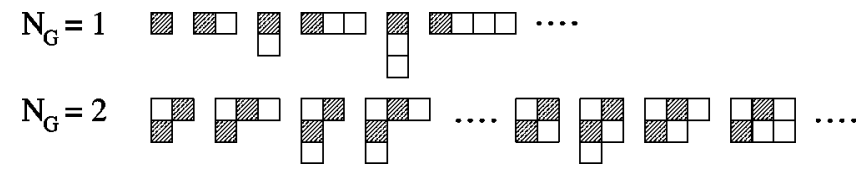

FIG. 4. YD's corresponding to $N_{G}=1$ and 2 .

number of boxes of $Y_{I}$ is the excitation energy of $I$ (in units of $2 d$ ) at $g=0$ for the equally spaced model.

\section{THERMODYNAMIC LIMIT}

As we explained in the previous section, by increasing $g$, $M-N_{G}$ of the energies $E_{\mu}$ become complex and arrange themselves into an arc which escapes to infinity for large $g$, while the remaining $N_{G}$ stay finite with their positions barely modified [see Figs. 1(b),1(c) and 2]. Following the procedure presented in Refs. 7 and 10-12 we take the large $N$ limit keeping $M / N, g$, and $N_{G}$ finite. In this limit the arc formed by the energies in the complex plane becomes dense, and allows for a continuous formulation. In particular, the GS corresponds to an arc $\Gamma_{I_{0}}$ in the complex energy plane, which in the $g \rightarrow \infty$ limit goes to infinity.

Excited states contain finite energies in addition to the arc. A given finite root $E_{\alpha}$ can be either real or complex. In the former case we shall call it a 1-string. In the latter case $E_{\alpha}^{*}$ is also a root, which together with $E_{\alpha}$ forms a 2-string [an example of such states can be seen in Fig. 3(b)]. There are also 3 -string formed by one real root and two complex ones, having approximately the same real part, and so on. In general $\left\{E_{\alpha}\right\}_{\alpha=1}^{N_{G}}$ is a combination of strings with several lengths. The remaining $M-N_{G}$ roots condense into an arc $\Gamma_{I}$, which is a slight perturbation of the GS arc $\Gamma_{I_{0}}$. In Fig. 2 we depict $\Gamma_{I_{0}}$ and $\Gamma_{I}$ for the two excited states $I_{1}$ and $I_{2}$ shown in Figs. 1(b) and 1(c).

Taking into account these considerations, and using the methods of Refs. 7 and 11, one can show in the large $N$ limit that the excitation energy of a Richardson state $I$ is given by

$$
E_{e x c}=\sum_{\alpha=1}^{N_{G}} \sqrt{\left(E_{\alpha}-\varepsilon_{0}\right)^{2}+\Delta^{2}}
$$

where $\varepsilon_{0}$ is twice the chemical potential, and the energies $E_{\alpha}$ satisfy the modified Gaudin equations

$$
0=\sum_{j=1}^{N} \frac{1}{R\left(\varepsilon_{j}\right)\left(\varepsilon_{j}-E_{\alpha}\right)}-\sum_{\beta \neq \alpha}^{N_{G}} \frac{2}{R\left(E_{\beta}\right)\left(E_{\beta}-E_{\alpha}\right)},
$$

with $R(E)=\sqrt{\left(E-\varepsilon_{0}\right)^{2}+\Delta^{2}}$. As $g \rightarrow \infty$ one has $\Delta \sim g \omega$ and Eqs. (7) become Eqs. (4).

The excitation energy given by Eq. (6) fits quite well the excitation energies of our prototype example $(N=40, M$ $=20$ ), as shown in Fig. 5. This also exhibits the linear behavior of the excitation energy for $g \rightarrow \infty$, i.e., $E_{\text {exc }} \sim N_{G} \Delta$ as stated in Sec. II, and in full agreement with the large $g$ behavior of Eq. (6). Thus we can extend our conjecture to the whole range of $g$. Namely, any excited state is composed of $N_{G}$ elementary excitations associated to the finite Gaudin 


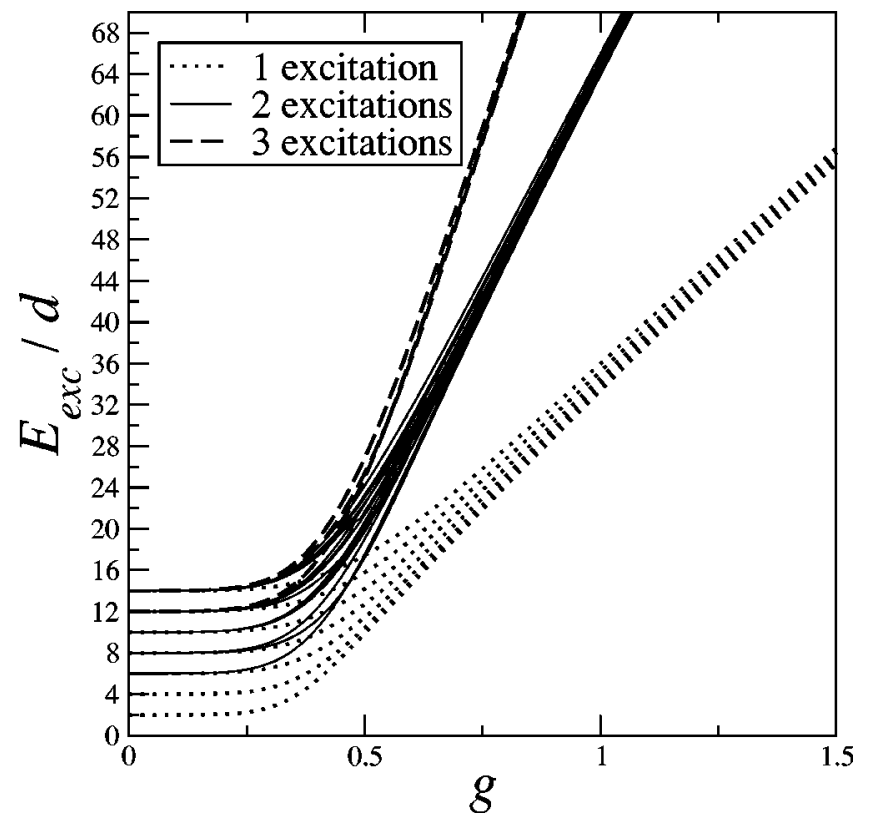

FIG. 5. Excitation energies $E_{\text {exc }}=E-E_{G S} \leqslant 14 d$ for $M=20$ pairs at half filling. There are $44=13+26+5$ states corresponding to $N_{G}=1,2$, and 3 , respectively. The particle-hole symmetry reduces these numbers to $25=7+15+3$.

energies. The Hilbert space spanned by these excitations has therefore a dimension $d_{N_{G}}=\left(\begin{array}{l}N_{G} \\ N\end{array}\right)-\left(\begin{array}{c}N_{G}-1 \\ N\end{array}\right)$. Hence, it is reasonable to call this new type of excitations Gaudin pairs or gaudinos.

In order to compare our results with the BCS standard solution let's consider the excitation energy given by a real Cooper pair in the Bogoliubov approach, which is given by $\sqrt{\varepsilon_{j}^{2}+\Delta^{2}}$ (notice that $\Delta \equiv 2 \Delta_{B C S}$ ), and spans a Hilbert space of dimension $\left(\begin{array}{c}N_{G} \\ N\end{array}\right)$. The standard Bogoliubov quasiparticle with an energy $\frac{1}{2} \sqrt{\varepsilon_{j}^{2}+\Delta^{2}}$ would have to be compared with excitations involving broken Cooper pairs. Since $E_{\alpha}$ in Eq. (6) lies between two energy levels, with $\varepsilon_{j+1}-\varepsilon_{j}=2 d$ $\sim 1 / N$ [e.g., in Fig. 1(b) $E_{20}(\infty)=0$ with $\varepsilon_{20}<E_{20}<\varepsilon_{21}$, $E_{\alpha}=\varepsilon_{j}+O(1 / N)$, and $d_{N_{G}}=\left(\begin{array}{c}N \\ N_{G}\end{array}\right)\left[1-N_{G} /\left(N-N_{G}+1\right)\right]$. Therefore, our theory is consistent within $O(1 / N)$ corrections, as is well known from the existing relation between a canonical and a grand canonical ensemble formulation in statistical physics. It is important to notice how the Bogoliubov excitations are uncorrelated with respect to the BCS ground state. We already pointed out that the correlation present in our formulation is lifted by choosing a shifted Fermi level $F L\left(\ell_{I}\right)$. This Fermi level is within a distance
$O(1 / N)$ from the original one. The selection of a new Fermi level leads to a new Fermi sea (with a different number of particles), allowing for a grand canonical formulation in a natural way.

In summary, our gaudinos will yield the same results as the BCS theory in the extreme $N=\infty$ limit, and will account for the exact corrections to the bulk results for the finite-size superconducting grains for all the physical observables and thermodynamic properties.

\section{CONCLUSIONS}

We have shown in this paper that the elementary excitations of the exactly solvable BCS model in the canonical ensemble can be explained by the Gaudin model and have no counterpart in the Bogoliubov picture of quasiparticles. Their peculiar dispersion relation and the unexpected counting properties, which are due to the correlated behavior of pairs and holes around the Fermi level, provide the exact finitesize corrections to the BCS bulk results, valid for large systems in the g.c. ensemble. These excitations, together with those obtained by breaking Cooper pairs, supply the complete spectrum of the canonical BCS model. A formula to compute the number of elementary excitations for any given state was also proposed.

We explained how the description in terms of gaudinos agrees with the Bogoliubov picture in the thermodynamic limit to leading order in $N$. In the case of broken pairs, which was not presented here, the mechanism is identical. It is of interest to study how the phase of the superconducting order parameter emerges from this fixed number of particles formulation. It will be intimately related to the possibility of choosing a shifted Fermi level in the large $N$ limit (which looses the correlation of the excitation), allowing the introduction of ground states with different number of pairs.

Although we used as an example a system of equally spaced levels, the results are more general, and apply to any distribution of levels. This assertion is based on numerical calculations considering broken Cooper pairs. In this case blocked levels are removed, and we are left with a nonequally spaced spectrum, obtaining again the same general results. In the case of nonconstant pairing we also expect the qualitative picture presented here to hold.

\section{ACKNOWLEDGMENTS}

We thank R. W. Richardson, C. Essebag, A. Di Lorenzo, A. Mastellone, L. Amico and A. Berkovich for discussions. This work has been supported by Grant No. BFM2000-1320C02-01/02.
${ }^{1}$ J. Bardeen, L.N. Cooper, and J.R. Schrieffer, Phys. Rev. 108, 1175 (1957).

${ }^{2}$ A. Bhor, B. Mottelson, and D. Pines, Phys. Rev. 110, 936 (1958).

${ }^{3}$ J. Dukelsky and G. Sierra, Phys. Rev. Lett. 83, 172 (1999); Phys. Rev. B 61, 12302 (2000).
${ }^{4}$ R.W. Richardson, Phys. Lett. 3, 277 (1963); R.W. Richardson and N. Sherman, Nucl. Phys. 52, 221 (1964).

${ }^{5}$ G. Sierra, J. Dukelsky, G.G. Dussel, J. von Delft, and F. Braun, Phys. Rev. B 61, 11890 (2000).

${ }^{6}$ J. von Delft and D.C. Ralph, Phys. Rep. 345, 61 (2001). 
${ }^{7}$ M. Gaudin, Modèles Exactament Résolus (Les Éditions de Physique, France, 1995).

${ }^{8}$ F. M. Goodman, P. de la Harpe, and V. F. R. Jones, Coxeter Graphs and Towers of Algebras (Springer-Verlag, New York, 1989).

${ }^{9}$ We thank A. Berkovich for providing us with an alternative proof based on counting RSOS paths.

${ }^{10}$ R.W. Richardson, J. Math. Phys. 18, 1802 (1977).

${ }^{11}$ J.M. Román, G. Sierra, J. Dukelsky, Nucl. Phys. B 634, 483 (2002).

${ }^{12}$ L. Amico, A. Di Lorenzo, A. Mastellone, A. Osterloh, and R. Raimondi, Ann. Phys. (N.Y.) 299, 228 (2002). 